\title{
Multicomponent Solvent-Free Synthesis, Antibacterial Evaluation and QSAR Study of 2-(Bis(benzylthio)methylene) malononitriles
}

\author{
Hamid Beyzaei, ${ }^{\star}$ Parviz Baranipour, Reza Aryan, Pouya Karimi, \\ Mahmood Sanchooli and Hojat Samareh Delarami \\ Department of Chemistry, Faculty of Science, University of Zabol, Zabol, Iran \\ *Corresponding author: E-mail: hbeyzaei@yahoo.com and hbeyzaei@uoz.ac.ir \\ Tel: +985431232186/ Fax: +985431232180
}

Received: 19-07-2018

\begin{abstract}
Multicomponent reaction of malononitrile, carbon disulphide and various benzyl halides was developed as an efficient strategy for the synthesis of 2-(bis(benzylthio)methylene)malononitrile derivatives via two different procedures: (a) in the presence of $\mathrm{K}_{2} \mathrm{CO}_{3}$ as a base in acetonitrile and (b) under solvent-free conditions in the presence of triethylamine. Higher yields with shorter reaction times were obtained from the latter procedure. Inhibitory activity of all derivatives was evaluated against 22 pathogenic bacteria including both Gram-negative and Gram-positive strains. Thioether $\mathbf{4 b}$ showed broad-spectrum antibacterial activities according to the antibiogram tests. DFT calculations (B3LYP/6-311++G ${ }^{* *}$ ) were performed to determine the type of drug-receptor interactions. It was found that reversible dipole-dipole forces play a key role in most interactions.
\end{abstract}

Keywords: Multicomponent reaction; solvent-free; thioether; antimicrobial activity; QSAR

\section{Introduction}

Organic compounds containing $\mathrm{C}-\mathrm{S}-\mathrm{C}$ bonds are known as sulfides or thioethers. These functional groups exist alone or alongside others in various pharmaceutical and biologically active molecules (Figure 1.). L-Methionine is a proteinogenic amino acid that plays an essential role in the growth of new blood vessels. It is a beneficial supplement to treat schizophrenia, asthma, alcoholism, Parkinson's disease, drug withdrawal, copper poisoning, allergies and depression diseases. Bithionol is a diarylthioether that is used against trematode and cestode infestations, especially in animals. ${ }^{1}$ It should be consumed with caution in human infections due to its photosensitizing effects on the skin. ${ }^{2}$ Cefotiam belongs to a class of

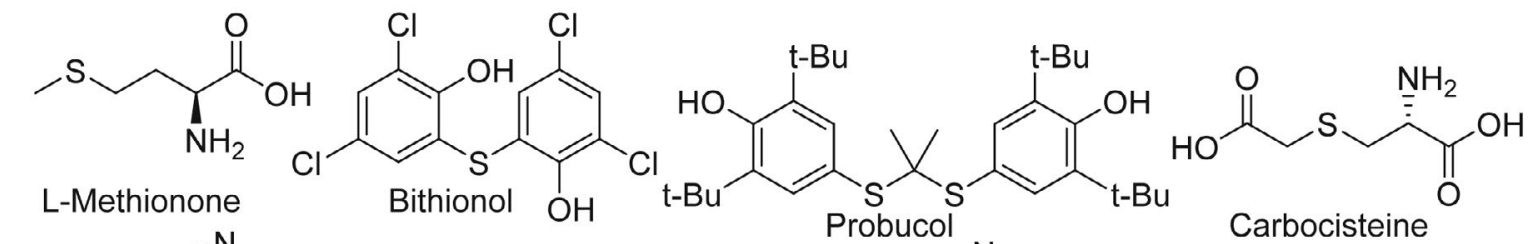<smiles>CC(C)(C)NCC(O)CSc1nc(-c2ccc(C(N)=O)s2)cs1</smiles>

Figure 1. Some approved thioether-based drugs. 
antibiotics known as cephalosporins. ${ }^{3}$ Butoconazole nitrate (Gynazole-1) is used for treatment of vulvovaginal yeast infections. ${ }^{4}$ Arotinolol is prescribed for the treatment of hypertension and essential tremor. ${ }^{5,6}$ Probucol as a potent antioxidant drug lowers the level of cholesterol (mainly LDL) in the blood. ${ }^{7}$ Carbocisteine ( $S$-carboxymethylcysteine), prepared by alkylation of cysteine using chloroacetic acid, improves the symptoms of chronic obstructive pulmonary disorder (COPD) and bronchiectasis. ${ }^{8}$

New methods and procedures were developed to synthesize organic sulfides. For this purpose, metal-catalyzed reactions, ${ }^{9-11}$ Sandmeyer and Leuckart reactions, ${ }^{12,13}$ coupling of thiols with Grignard reagents in the presence of $\mathrm{N}$-chlorosuccinimide, ${ }^{14}$ photocatalytically initiated thiol-ene reaction, ${ }^{15}$ free radical displacement on alkynes, ${ }^{16}$ regioselective conjugate addition of thiols to acyclic $\alpha, \beta, \gamma, \delta$-unsaturated dienones, ${ }^{17}$ in situ nucleophilic substitution of aryl bromides with potassium iodomethyltrifluoroborates, ${ }^{18}$ displacement reaction of halogens by sulfur, ${ }^{19}$ Stevens rearrangement of thioethers with arynes, ${ }^{20}$ and synthesis of thiiranes via reaction of epoxides with thiourea in $\mathrm{DES}^{21}$ were proposed by researchers. Alkylation of thiols or their salts is a well-developed method for the preparation of thioether derivatives. ${ }^{22,23}$ Furthermore, these compounds were successfully synthesized via a variety of one-pot multistep ${ }^{24-26}$ and multicomponent ${ }^{27,28}$ alkylation reactions.

Theoretical studies can guide chemists to design and discover new medicines as well as to predict their action mechanisms. Some thioether-ester crown ethers were synthesized as potential inhibitors of Klebsiella pneumoniae. ${ }^{29}$ QSAR calculations including Moriguchi octanol-water partition coefficient, polar surface area, hydrophilic factor, Ghose-Crippen molar refractivity, unsaturation index and weighted holistic invariant molecular descriptors were in compliance with the MIC value of the synthetic compounds. ${ }^{29}$ Antiplatelet activities of some synthesized 2-(arylmethylthio)-3-phenylquinazolin-4-ones were evaluated on ADP and arachidonic acid-induced platelet aggregation in human plasma. ${ }^{30}$ Theoretical calculations showed a fairly parabolic correlation between $\mathrm{IC}_{50}$ values of derivatives and their related molecular volume and surface area. Recently, a connection was determined between thioether pleuromutilin derivatives and bacterial 50S ribosomal protein L3 using 3D-QSAR and Topomer CoMFA analysis and ADMET prediction. ${ }^{31}$

In this project, some 2-(bis(benzylthio)methylene) malononitrile derivatives were synthesized via two pathways, namely in the presence of a solvent and under solvent-free conditions. Inhibitory properties of these symmetric thioethers were assessed against different genera of bacterial pathogens. QSAR studies were applied to predict the correlation between biological activities of synthesized thioethers and physicochemical descriptors.

\section{Experimental}

\section{1. Chemicals}

All yields refer to isolated products. Melting points were recorded on a Kruss type KSP1N melting point apparatus and are corrected. The reaction progress was monitored by aluminium TLC plates pre-coated with silica gel with fluorescent indicator F254 using $n$-hexane/ethyl acetate $(9: 1, \mathrm{v} / \mathrm{v})$ as the desired mobile phase. The resulted TLC plates were visualized under UV radiation $(254 \mathrm{~nm})$. The IR spectra of the products were recorded on a Bruker Tensor-27 FT-IR spectrometer using $\mathrm{KBr}$ disks. The ${ }^{1} \mathrm{H}$ and ${ }^{13} \mathrm{C}$ NMR spectra of compounds in DMSO- $d_{6}$ or $\mathrm{CDCl}_{3}$ were recorded on a Bruker FT-NMR Ultra Shield-400 spectrometer (400 and $100 \mathrm{MHz}$, respectively). CHNS/O analyses were performed on a Thermo Finnigan Flash EA microanalyzer. Initial bacterial or fungal suspensions were adjusted with a Jenway $6405 \mathrm{UV} / \mathrm{Vis}$ spectrophotometer.

\section{1. 1. General Procedure for the Synthesis of Thioethers 4a-h}

\section{1. 1. 1. The Classical Conditions}

$0.66 \mathrm{~g}$ malononitrile $(\mathbf{1})(10 \mathrm{mmol}), 0.76 \mathrm{~g}$ carbon disulfide (2) $(10 \mathrm{mmol}), 2.76 \mathrm{~g}$ potassium carbonate $(20 \mathrm{mmol})$ and benzylhalides $\mathbf{3 a}-\mathbf{h}(20 \mathrm{mmol})$ in $20 \mathrm{~mL}$ acetonitrile were mixed well. The mixture was heated under reflux for $5-9 \mathrm{~h}$. The end of the reaction was indicated by TLC. The reaction mixture was cooled to room temperature. The contents were added to $20 \mathrm{~mL}$ water and extracted with diethyl ether $(2 \times 10 \mathrm{~mL})$. The extract was washed with water $(3 \times 15$ $\mathrm{mL}$ ), and the organic phase was separated, dried over anhydrous $\mathrm{MgSO}_{4}$, filtered and concentrated under reduced pressure. The solid residues were recrystallized from different mixtures of ethanol and water to give colorless needles.

\section{1. 1. 2. The Solvent-Free Conditions}

$0.66 \mathrm{~g}$ malononitrile $(\mathbf{1})(10 \mathrm{mmol}), 0.76 \mathrm{~g}$ carbon disulfide (2) $(10 \mathrm{mmol}), 2.02 \mathrm{~g}$ triethylamine $(20 \mathrm{mmol})$ and benzylhalides $\mathbf{3 a}-\mathbf{h}(20 \mathrm{mmol})$ were vigorously stirred to form a macroscopically homogeneous mixture. The mixture was heated at $80{ }^{\circ} \mathrm{C}$ for $4-7.5 \mathrm{~h}$. The progress of the reaction was monitored by TLC. After cooling to room temperature, the contents were added to $20 \mathrm{~mL}$ water. The procedures of extraction and purification proceeded similarly to the classical conditions.

2. 1. 1. 3. 2-(Bis(benzylthio)methylene)malononitrile (4a). Light orang needles; m.p. 87-88 ${ }^{\circ} \mathrm{C}$ (Ref. ${ }^{32}$ 85-86 $\left.{ }^{\circ} \mathrm{C}\right)$; IR $(\mathrm{KBr})$ v 2924, $2214(\mathrm{C} \equiv \mathrm{N}), 1588,1502,1449$ $\left(\mathrm{CH}_{2}\right), 1248,1091,831,711 \mathrm{~cm}^{-1}$.

\subsubsection{2-(Bis((2-nitrobenzyl)thio) methylene) malononitrile (4b). \\ Dark brown needles; m.p. $82-84^{\circ} \mathrm{C}$; IR $(\mathrm{KBr}) v 2925$, 2360, $2176(\mathrm{C} \equiv \mathrm{N}), 1635,1558$ (asymmetric $\mathrm{NO}_{2}$ stretch-}


ing), $1448\left(\mathrm{CH}_{2}\right), 1312$ (symmetric $\mathrm{NO}_{2}$ stretching), 1236, $1008,833,703 \mathrm{~cm}^{-1} ;{ }^{1} \mathrm{H}$ NMR $\left(400 \mathrm{MHz}, \mathrm{CDCl}_{3}\right) \delta 7.38(\mathrm{~s}$, $\left.2 \mathrm{H}, \mathrm{H}-3^{\prime}\right), 7.26-7.30$ (m, 4H, $\left.2 \mathrm{H}-5^{\prime}, 6^{\prime}\right), 7.20$ (m, 2H, 2 $\mathrm{H}-4$ '), 3.74 (s, $\left.4 \mathrm{H}, 2 \mathrm{CH}_{2}\right)$ ppm; ${ }^{13} \mathrm{C}$ NMR $(100 \mathrm{MHz}$, $\left.\mathrm{CDCl}_{3}\right) \delta 33.1\left(2 \mathrm{CH}_{2}\right), 119.4(\mathrm{NC}-\underline{\mathrm{C}}=\mathrm{C}), 121.4(2 \mathrm{C} \equiv \mathrm{N})$, 127.1 (2 C-6'), 129.5 (2 C-4'), 130.2 (2 C-5'), 131.3 (2 C-3'), 134.8 (2 C-1'), 147.3 (2 C-2'), 166.2 (NC-C=ㅡ) ppm. Anal. Calcd. for $\mathrm{C}_{18} \mathrm{H}_{12} \mathrm{~N}_{4} \mathrm{O}_{4} \mathrm{~S}_{2}: \mathrm{C}, 52.42 ; \mathrm{H}, 2.93 ; \mathrm{N}, 13.58$; $\mathrm{S}$, 15.55. Found: C, 52.37; H, 2.94; N, 13.62; S, 15.50 .

\section{1. 1. 5. 2-(Bis((4-nitrobenzyl)thio)methylene) malononitrile (4c).}

Light brown needles; m.p. 93-94 ${ }^{\circ} \mathrm{C}$; IR $(\mathrm{KBr}) v 2924$, 2360, $2189(\mathrm{C} \equiv \mathrm{N}), 1635,1558$ (asymmetric $\mathrm{NO}_{2}$ stretching), $1456\left(\mathrm{CH}_{2}\right), 1345$ (symmetric $\mathrm{NO}_{2}$ stretching), 1312, $1236,1008,833,703 \mathrm{~cm}^{-1} ;{ }^{1} \mathrm{H} \mathrm{NMR}\left(400 \mathrm{MHz}, \mathrm{DMSO}-d_{6}\right)$ $\delta 8.10\left(\mathrm{~d}, J=7.3 \mathrm{~Hz}, 4 \mathrm{H}, 2 \mathrm{H}-3^{\prime}, 5^{\prime}\right), 7.50(\mathrm{~d}, J=7.3 \mathrm{~Hz}, 4 \mathrm{H}$, $2 \mathrm{H}-2$ ',6'), 3.79 (s, 4H, $\left.2 \mathrm{CH}_{2}\right)$ ppm; ${ }^{13} \mathrm{C} \mathrm{NMR}(100 \mathrm{MHz}$, DMSO- $\left.d_{6}\right) \delta 34.9\left(2 \mathrm{CH}_{2}\right), 120.6(\mathrm{NC}-\mathrm{C}=\mathrm{C}), 123.9(2$

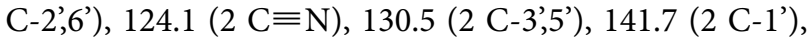
146.9 (2 C-4'), $170.1(\mathrm{NC}-\mathrm{C}=\underline{\mathrm{C}}) \mathrm{ppm}$. Anal. Calcd. for $\mathrm{C}_{18} \mathrm{H}_{12} \mathrm{~N}_{4} \mathrm{O}_{4} \mathrm{~S}_{2}$ : C, 52.42; H, 2.93; N, 13.58; $\mathrm{S}, 15.55$. Found: C, 52.36; H, 2.92; N, 13.63; S, 15.53 .

\section{1. 1. 6. 2-(Bis((2,4-dinitrobenzyl)thio)methylene) malononitrile (4d).}

Dark brown needles; m.p. $157-159^{\circ} \mathrm{C}$ (decomp.); IR $(\mathrm{KBr}) v 2924,2360,2218(\mathrm{C} \equiv \mathrm{N}), 1604,1532$ (asymmetric $\mathrm{NO}_{2}$ stretching), $1457\left(\mathrm{CH}_{2}\right), 1346$ (symmetric $\mathrm{NO}_{2}$ stretching), 1067, 853, $777 \mathrm{~cm}^{-1} ;{ }^{1} \mathrm{H}$ NMR $(400 \mathrm{MHz}$, DMSO- $\left.d_{6}\right) \delta 8.77\left(\mathrm{~s}, 2 \mathrm{H}, 2 \mathrm{H}-3^{\prime}\right), 8.58(\mathrm{~d}, J=8.2 \mathrm{~Hz}, 2 \mathrm{H}, 2$ H-5'), 8.18 (d, J = 8.2 Hz, 2H, $2 \mathrm{H}-6$ '), 3.48 (s, 4H, $2 \mathrm{CH}_{2}$ ) ppm; ${ }^{13} \mathrm{C}$ NMR $\left(100 \mathrm{MHz}, \mathrm{DMSO}-d_{6}\right) \delta 30.2\left(2 \mathrm{CH}_{2}\right)$, $120.8(2 \mathrm{C} \equiv \mathrm{N}), 127.7(\mathrm{NC}-\underline{\mathrm{C}}=\mathrm{C}), 128.4\left(2 \mathrm{C}-3^{\prime}\right), 130.2(2$ C-6'), 130.7 (2 C-5'), 137.0 (2 C-1'), 147.4 (2 C-4'), 148.2 (2 C-2'), $168.2 \quad(\mathrm{NC}-\mathrm{C}=\underline{\mathrm{C}})$ ppm. Anal. Calcd. for $\mathrm{C}_{18} \mathrm{H}_{10} \mathrm{~N}_{6} \mathrm{O}_{8} \mathrm{~S}_{2}$ : C, 43.03; H, 2.01; N, 16.73; S, 12.76. Found: C, $43.09 ; \mathrm{H}, 2.02 ; \mathrm{N}, 16.68 ; \mathrm{S}, 12.80 \%$.

\section{1. 1. 2-(Bis((2-chlorobenzyl)thio)methylene) malononitrile (4e).}

Dark brown needles; m.p. 87-88 ${ }^{\circ} \mathrm{C}$; IR (KBr) v 2922, $2224(\mathrm{C} \equiv \mathrm{N}), 1746,1572,1444\left(\mathrm{CH}_{2}\right), 1377,1237,1162$, $1052(\mathrm{C}-\mathrm{Cl}), 823,760 \mathrm{~cm}^{-1} ;{ }^{1} \mathrm{H}$ NMR $\left(400 \mathrm{MHz}, \mathrm{CDCl}_{3}\right) \delta$ $7.36\left(\mathrm{~m}, 2 \mathrm{H}, 2 \mathrm{H}-3^{\prime}\right), 7.22\left(\mathrm{~m}, 6 \mathrm{H}, 2 \mathrm{H}-4^{\prime}, 5^{\prime}, 6^{\prime}\right), 3.76(\mathrm{~s}, 4 \mathrm{H}$, $\left.2 \mathrm{CH}_{2}\right) \mathrm{ppm} ;{ }^{13} \mathrm{C} \mathrm{NMR}\left(100 \mathrm{MHz}, \mathrm{CDCl}_{3}\right) \delta 41.0\left(2 \mathrm{CH}_{2}\right)$, $121.8(2 \mathrm{C} \equiv \mathrm{N}), 126.7(\mathrm{NC}-\underline{\mathrm{C}}=\mathrm{C}), 128.4\left(2 \mathrm{C}-4^{\prime}\right), 128.9$ (2 C-3'), 129.7 (2 C-5'), 130.7 (2 C-2'), 130.9 (2 C-6'), 131.5 (2 C-1'), 162.4 (NC-C=) ppm. Anal. Calcd. for $\mathrm{C}_{18} \mathrm{H}_{12} \mathrm{Cl}_{2} \mathrm{~N}_{2} \mathrm{~S} 2$ : C, 55.25; H, 3.09; N, 7.16; S, 16.39. Found: C, 55.27; H, 3.08; N, 7.14; S, 16.43 .

\section{1. 1. 8. 2-(Bis((2,4-dichlorobenzyl)thio $)$ methylene $)$ malononitrile (4f). \\ Dark brown needles; m.p. $88-90^{\circ} \mathrm{C}$; IR (KBr) v 2926, $2220(\mathrm{C} \equiv \mathrm{N}), 1739,1587,1453\left(\mathrm{CH}_{2}\right), 1237,1050(\mathrm{C}-\mathrm{Cl})$,}

$867,727 \mathrm{~cm}^{-1}$; ${ }^{1} \mathrm{H}$ NMR $\left(400 \mathrm{MHz}, \mathrm{DMSO}-d_{6}\right) \delta 7.56(\mathrm{~s}$, 2H, $\left.2 \mathrm{H}-3^{\prime}\right), 7.32-7.45$ (m, 4H, $\left.2 \mathrm{H}-5^{\prime}, 6^{\prime}\right), 3.78$ (s, 4H, 2 $\left.\mathrm{CH}_{2}\right)$ ppm; ${ }^{13} \mathrm{C}$ NMR $\left(100 \mathrm{MHz}, \mathrm{DMSO}-d_{6}\right) \delta 33.1(2$ $\left.\mathrm{CH}_{2}\right), 122.4(2 \mathrm{C} \equiv \mathrm{N}), 125.9(\mathrm{NC}-\underline{\mathrm{C}}=\mathrm{C}), 127.7\left(2 \mathrm{C}-5^{\prime}\right)$, 129.3 (2 C-3'), 132.6 (2 C-1'), 133.5 (2 C-6'), 134.4 (2 C-4'), 135.2 (2 C-2'), $163.7(\mathrm{NC}-\mathrm{C}=\underline{\mathrm{C}}) \mathrm{ppm}$. Anal. Calcd. for $\mathrm{C}_{18} \mathrm{H}_{10} \mathrm{Cl}_{4} \mathrm{~N}_{2} \mathrm{~S}_{2}: \mathrm{C}, 46.98 ; \mathrm{H}, 2.19 ; \mathrm{N}, 6.09 ; \mathrm{S}, 13.93$. Found: C, 47.04; H, 2.18; N, 6.08; S, 13.89 .

\section{1. 1. 9. 2-(Bis((2-cyanobenzyl)thio)methylene) malononitrile $(4 \mathrm{~g})$.}

Dark yellow needles; m.p. $94-96^{\circ} \mathrm{C}$; IR (KBr) $v 2925$, $2176(\mathrm{C} \equiv \mathrm{N}), 1652,1488\left(\mathrm{CH}_{2}\right), 1373,1008,869,703$ $\mathrm{cm}^{-1} ;{ }^{1} \mathrm{H}$ NMR $\left(400 \mathrm{MHz}, \mathrm{DMSO}-d_{6}\right) \delta 7.67(\mathrm{~m}, 6 \mathrm{H}$, $\left.2 \mathrm{H}-3^{\prime}, 4^{\prime}, 5^{\prime}\right), 7.46$ (m, 2H, $\left.2 \mathrm{H}-6^{\prime}\right), 3.81\left(\mathrm{~s}, 4 \mathrm{H}, 2 \mathrm{CH}_{2}\right) \mathrm{ppm}$; ${ }^{13} \mathrm{C}$ NMR (100 MHz, DMSO- $\left.d_{6}\right) \delta 34.9\left(2 \mathrm{CH}_{2}\right), 115.4$ (2 C-2'), 120.6 (NC- $\underline{C}=\mathrm{C}), 122.1$ (2 C-4'), 123.9 (2 C-6'), $124.1(2 \mathrm{C} \equiv \mathrm{N}), 124.7\left(\mathrm{C} \equiv \mathrm{N}-2^{\prime}\right), 129.2\left(2 \mathrm{C}-3^{\prime}\right), 130.5$ (2 C-5'), 146.9 (2 C-1'), 170.1 (NC-C=ㅅ) ppm. Anal. Calcd. for $\mathrm{C}_{20} \mathrm{H}_{12} \mathrm{~N}_{4} \mathrm{~S}_{2}: \mathrm{C}, 64.49 ; \mathrm{H}, 3.25 ; \mathrm{N}, 15.04 ; \mathrm{S}, 17.22$. Found: C, 64.52; H, 3.26; N, 15.02; S, 17.20.

\section{1. 1. 10.2-(Bis $(($ perfluorophenyl)methyl)thio $)$ methylene)malononitrile (4h).}

Cream needles; m.p. $97-99^{\circ} \mathrm{C}$; IR (KBr) v 2927, 2199 $(\mathrm{C} \equiv \mathrm{N}), 1715,1522,1474\left(\mathrm{CH}_{2}\right), 1311,1125$ (C-F), 993, $965,752 \mathrm{~cm}^{-1} ;{ }^{1} \mathrm{H}$ NMR $\left(400 \mathrm{MHz}, \mathrm{DMSO}-d_{6}\right) \delta 3.92(\mathrm{~s}, 2$ $\left.\mathrm{CH}_{2}\right) \mathrm{ppm} ;{ }^{13} \mathrm{C}$ NMR $\left(100 \mathrm{MHz}, \mathrm{DMSO}-d_{6}\right) \delta 23.2$ $\left(2 \mathrm{CH}_{2}\right), 112.9(\mathrm{NC}-\mathrm{C}=\mathrm{C}), 115.5(2 \mathrm{C} \equiv \mathrm{N}), 118.0\left(2 \mathrm{C}-1^{\prime}\right)$, 139.3 (2 C-2',6'), 143.0 (2 C-4'), 146.9 (2 C-3',5'), 162.1 (NC$\mathrm{C}=\underline{\mathrm{C}}$ ) ppm. Anal. Calcd. for $\mathrm{C}_{18} \mathrm{H}_{4} \mathrm{~F}_{10} \mathrm{~N}_{2} \mathrm{~S}_{2}: \mathrm{C}, 43.04 ; \mathrm{H}, 0.80$; N, 5.58; S, 12.76. Found: C, 43.01; H, 0.80; N, 5.60; S, 12.78.

\section{2. In vitro Antibacterial Activity}

Gram-negative bacterial strains including Acinetobacter baumannii (PTCC 1855), Pseudomonas aeruginosa (PTCC 1310), Klebsiella pneumoniae (PTCC 1290), Escherichia coli (PTCC 1399), Shigella flexneri (PTCC 1234), Shigella dysenteriae (PTCC 1188), Proteus mirabilis (PTCC 1776), Proteus vulgaris (PTCC 1079), Salmonella enterica subsp. enterica (PTCC 1709) and Salmonella typhi (PTCC 1609), and Gram-positive bacterial strains including Enterococcus faecalis (PTCC 1778), Streptococcus pyogenes (PTCC 1447), Streptococcus agalactiae (PTCC 1768), Streptococcus equinus (PTCC 1445), Streptococcus pneumoniae (PTCC 1240), Listeria monocytogenes (PTCC 1297), Staphylococcus aureus (PTCC 1189), Staphylococcus epidermidis (PTCC 1435), Bacillus cereus (PTCC 1665), Bacillus subtilis subsp. spizizenii (PTCC 1023), Bacillus thuringiensis subsp. kurstaki (PTCC 1494), Rhodococcus equi (PTCC 1633) were prepared from the Persian Type Culture Collection (PTCC), Karaj, Iran. IZD, MIC and $\mathrm{MBC}$ values were determined by using broth disk diffusion, microdilution and time-kill methods, according to CLSI (Clinical and Laboratory Standards Institute) guide- 
lines M07-A9, M26-A and M02-A11. ${ }^{33}$ The stock solutions of all derivatives and antibiotics were respectively prepared in $10 \%$ DMSO and double-distilled water at concentrations of 10240 and $17.6 \mathrm{mg} \mathrm{mL}^{-1}$. The IZD values were measured at these initial concentrations. All antibiogram tests were performed at least three times independently, and the results are reported as mean values.

\section{3. Computational Details}

All geometries were optimized at the B3LYP level of density functional theory using the $6-31+G(d, p)$ basis set, and no molecular symmetry constraint was considered. ${ }^{34}$ All calculations were performed using Gaussian 09 program package at $298.15 \mathrm{~K}$ and $1 \mathrm{~atm}$. Vibrational frequency analyses were also performed at the same level to ensure that the optimized structures are local minima. The electron density distribution was analyzed by the atoms in molecules (AIM) and the natural bond orbital (NBO) methods on the wave functions obtained at the B3LY$\mathrm{P} / 6-31+\mathrm{G}^{* *}$ level of theory by AIM2000 and NBO 3.1 programs, respectively. ${ }^{35,36}$

\section{Results and Discussion}

\section{1. Synthesis and Spectroscopic Characterization of Thioethers $4 a-h$}

2-(Bis(benzylthio)methylene)malononitriles $\quad \mathbf{4 a}-\mathbf{h}$ were prepared under two different conditions (Scheme 1). Malononitrile (1), carbon disulfide (2) and benzyl halides 3a-h were simultaneously reacted under classical conditions and in the presence of potassium carbonate and acetonitrile as reaction promoter and solvent. However, shorter reaction times were necessary and higher product yields were obtained under solvent-free conditions using triethylamine as the base (Table 1). As expected, the probability of efficient interactions between the reactants has increased in the absence of a solvent.

Chemical structures of the molecules $4 \mathbf{a}-\mathbf{h}$ were determined according to their spectral data and elemental analyses. In NMR spectra, hydrogens and carbons of the methylene groups appeared respectively within 3.48-3.92 and 23.17-41.04 ppm. Two carbons of olefin bonds were observed in low- and high-field regions of ${ }^{13} \mathrm{C}-\mathrm{NMR}$ spectra. In addition, absorption bands associated with $\mathrm{C} \equiv \mathrm{N}$ stretching vibrations were assigned to signals at 2176-2224 $\mathrm{cm}^{-1}$.

\section{2. Antibacterial Evaluation of the Synthesized Compounds}

Inhibitory activities of all synthesized thioethers were evaluated against 22 important Gram-positive and Gram-negative bacterial pathogens. The biological properties of compounds were compared with those of gentamicin antibiotic, and are reported as IZD (the inhibition zone diameter), MIC (the minimum inhibitory concentration) and $\mathrm{MBC}$ (the minimum bactericidal concentration) values in Tables 2 and 3 . Thioether $\mathbf{4 b}$ containing two 2-nitrobenzyl substituents could inhibit the growth of all tested bacteria. This broad-spectrum antibacterial agent was the only effective sulfide on Proteus vulgaris, Streptococcus pneumoniae and Shigella dysenteriae species. The shift of the nitro groups to the para-positions in compound $4 \mathrm{c}$ led to a significant reduction in antibacterial effects, this compound was ineffective on all tested Gram-negative strains. These effects did not change remarkably by the introduction of the second nitro group in compound 4d. However, a similar change in compounds $\mathbf{4 e}, \mathbf{f}$ resulted in the expansion of spectrum of action.

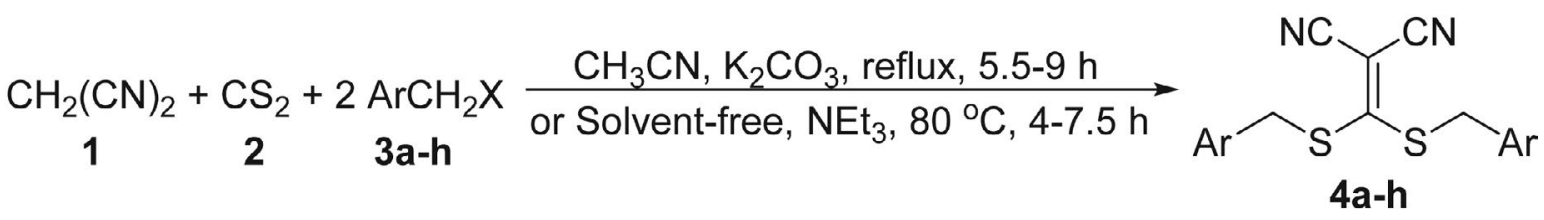

Scheme 1. Total synthesis of thioethers $4 \mathbf{a}-\mathbf{h}$.

Table 1. Multicomponent synthesis of thioethers $\mathbf{4 a - h}$ in acetonitrile or under solvent-free conditions.

\begin{tabular}{lllccccc}
\hline Entry & Ar & $\mathbf{X}$ & Products & \multicolumn{2}{c}{ Time/h } & \multicolumn{2}{c}{ Yield/\% } \\
& & & & Acetonitrile & Solvent-free & Acetonitrile & Solvent-free \\
\hline 1 & $\mathrm{C}_{6} \mathrm{H}_{5}$ & $\mathrm{Cl}$ & $\mathbf{4 a}$ & 7 & 5 & 90 & 96 \\
2 & $2-\mathrm{O}_{2} \mathrm{~N}-\mathrm{C}_{6} \mathrm{H}_{4}$ & $\mathrm{Cl}$ & $\mathbf{4 b}$ & 5 & 4 & 92 & 98 \\
3 & $4-\mathrm{O}_{2} \mathrm{~N}_{-}-\mathrm{C}_{6} \mathrm{H}_{4}$ & $\mathrm{Br}$ & $\mathbf{4 c}$ & 6.5 & 5.5 & 88 & 95 \\
4 & $2,4-\left(\mathrm{O}_{2} \mathrm{~N}\right)_{2}-\mathrm{C}_{6} \mathrm{H}_{3}$ & $\mathrm{Cl}$ & $\mathbf{4 d}$ & 9 & 7.5 & 81 & 89 \\
5 & $2-\mathrm{Cl}-\mathrm{C}_{6} \mathrm{H}_{4}$ & $\mathrm{Cl}$ & $\mathbf{4 e}$ & 6 & 4 & 91 & 97 \\
6 & $2,4-(\mathrm{Cl})_{2} \mathrm{C}_{6} \mathrm{H}_{3}$ & $\mathrm{Cl}$ & $\mathbf{4 f}$ & 5.5 & 4 & 89 & 98 \\
7 & $2-\mathrm{NC}-\mathrm{C}_{6} \mathrm{H}_{4}$ & $\mathrm{Br}$ & $\mathbf{4 g}$ & 6 & 4 & 87 & 93 \\
8 & $\mathrm{C}_{6} \mathrm{~F}_{5}$ & $\mathrm{Br}$ & $\mathbf{4 h}$ & 7 & 5.5 & 86 & 94 \\
\hline
\end{tabular}


Table 2. Inhibitory activities of thioethers $\mathbf{4 a - h}$ against Gram-negative pathogenic bacteria.

\begin{tabular}{|c|c|c|c|c|c|c|c|c|c|c|}
\hline Bacteria & & $\begin{array}{l}\text { Prod } \\
4 a\end{array}$ & ts $4 b$ & $4 c$ & $4 d$ & $4 e$ & $4 f$ & $4 \mathrm{~g}$ & $4 h$ & $\begin{array}{l}\text { Antibiotic } \\
\text { Gentamicin }\end{array}$ \\
\hline \multirow[t]{3}{*}{1855} & IZD & - & 22.76 & - & - & 13.10 & 15.40 & 12.11 & - & 19.63 \\
\hline & MIC & - & 64 & - & - & 512 & 128 & 512 & - & 16 \\
\hline & $\mathrm{MBC}$ & - & 128 & - & - & 1024 & 256 & 1024 & - & 32 \\
\hline \multirow[t]{3}{*}{1310} & IZD & - & 12.56 & - & - & - & - & 11.65 & - & 25.90 \\
\hline & MIC & - & 512 & - & - & - & - & 512 & - & 0.063 \\
\hline & $\mathrm{MBC}$ & - & 1024 & - & - & - & - & 1024 & - & 0.063 \\
\hline \multirow[t]{3}{*}{1290} & IZD & - & 13.11 & - & - & - & - & 14.56 & - & 21.78 \\
\hline & MIC & - & 512 & - & - & - & - & 256 & - & 4 \\
\hline & $\mathrm{MBC}$ & - & 1024 & - & - & - & - & 512 & - & 4 \\
\hline \multirow[t]{3}{*}{1399} & IZD & - & 11.80 & - & - & 10.12 & 8.90 & - & - & 23.60 \\
\hline & MIC & - & 256 & - & - & 512 & 1024 & - & - & 8 \\
\hline & $\mathrm{MBC}$ & - & 512 & - & - & 1024 & 2048 & - & - & 8 \\
\hline \multirow[t]{3}{*}{1234} & IZD & 7.39 & 30.45 & - & 9.80 & 6.89 & 9.90 & 16.11 & 10.62 & 19.46 \\
\hline & MIC & 512 & 16 & - & 256 & 1024 & 256 & 128 & 256 & 2 \\
\hline & $\mathrm{MBC}$ & 1024 & 64 & - & 512 & 2048 & 512 & 256 & 512 & 8 \\
\hline \multirow[t]{3}{*}{1188} & IZD & - & 12.65 & - & - & - & - & - & - & 21.16 \\
\hline & MIC & - & 128 & - & - & - & - & - & - & 0.031 \\
\hline & $\mathrm{MBC}$ & - & 256 & - & - & - & - & - & - & 0.063 \\
\hline \multirow[t]{3}{*}{1776} & IZD & 11.60 & 10.40 & - & - & - & - & 10.80 & - & 21.82 \\
\hline & MIC & 512 & 512 & - & - & - & - & 512 & - & 0.063 \\
\hline & $\mathrm{MBC}$ & 1024 & 1024 & - & - & - & - & 1024 & - & 1 \\
\hline \multirow[t]{3}{*}{1079} & IZD & - & 7.80 & - & - & - & - & - & - & 30.26 \\
\hline & MIC & - & 512 & - & - & - & - & - & - & 4 \\
\hline & $\mathrm{MBC}$ & - & 1024 & - & - & - & - & - & - & 4 \\
\hline \multirow[t]{3}{*}{1709} & IZD & - & 21.67 & - & - & - & - & 13.34 & - & 23.84 \\
\hline & MIC & - & 64 & - & - & - & - & 256 & - & 8 \\
\hline & $\mathrm{MBC}$ & - & 128 & - & - & - & - & 512 & - & 8 \\
\hline \multirow[t]{3}{*}{1609} & IZD & - & 21.80 & - & - & - & - & 11.67 & - & 21.29 \\
\hline & MIC & - & 64 & - & - & - & - & 512 & - & 0.063 \\
\hline & $\mathrm{MBC}$ & - & 128 & - & - & - & - & 1024 & - & 0.125 \\
\hline
\end{tabular}

QSAR studies were used to correlate biological activities of compounds into their physicochemical parameters. It is well known that the antibacterial activity of antibiotics is related to intermolecular interactions between the drug and receptor. Identification of the type of forces involved is necessary to adequately explore drug-receptor interactions. Intermolecular interactions are mainly divided into reversible dipole-dipole forces, weak London dispersion forces and stronger irreversible covalent bonding. The challenge of determining these interactions particularly in antibacterial agents has been the subject of a vast amount of both theoretical and experimental studies.

In this investigation, the IZD values of synthesized thioethers $\mathbf{4 a}-\mathbf{h}$ were computationally related to their physicochemical descriptors. For this purpose, all three classes of intermolecular interactions have been considered. Figure 2 shows the typical structure of studied ligands.

$\mathrm{S}_{\mathrm{N}} 2$, Schiff base formation and Michael addition are chemical interactions involved in drug-receptor complexes. Covalent bonds can be usually formed via nucleophilic attack on electron-deficient sites of biological macromolecules. The Pearson's Hard and Soft, Acids and Bases (HSAB)

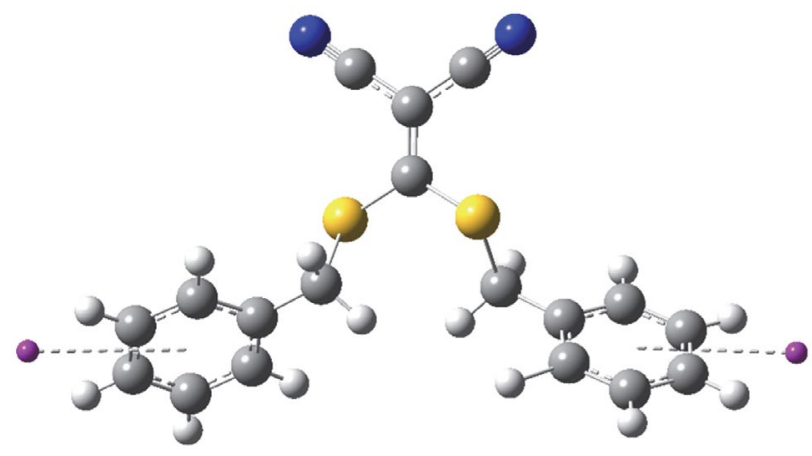

Figure 2. Typical structure of thioethers $\mathbf{4 a}-\mathbf{h}$.

theory predicts a significant degree of selectivity that occurs in such nucleophile-electrophile interactions. ${ }^{37-39}$ Accordingly, important electronic descriptors including hardness (HD), softness (SOF), electronegativity (EN) and electrophilicity $(\mathrm{EPH})$, which are obtained from energies of HOMO and LUMO, were used to characterize irreversible covalent interactions. They, respectively, describe stability, reactivity, electron affinity and a measure of energy lowering due to maximal electron flow between the donor and acceptor. ${ }^{40}$ They can be calculated as: ${ }^{41}$ 
Table 3. Inhibitory activities of thioethers $4 \mathbf{a}-\mathbf{h}$ against Gram-positive pathogenic bacteria.

\begin{tabular}{|c|c|c|c|c|c|c|c|c|c|c|}
\hline Bacteria & Prod & $\begin{array}{l}\text { ucts } \\
\qquad 4 a\end{array}$ & $4 b$ & $4 c$ & $4 d$ & $4 e$ & $4 f$ & $4 g$ & $4 h$ & $\begin{array}{l}\text { Antibiotic } \\
\text { Gentamicin }\end{array}$ \\
\hline \multirow[t]{3}{*}{1778} & $\mathrm{IZD}^{\mathrm{a}}$ & - & 13.90 & - & - & 14.80 & - & - & 14.80 & 15.64 \\
\hline & $\mathrm{MIC}^{\mathrm{b}}$ & - & 128 & - & - & 128 & - & - & 128 & 0.5 \\
\hline & $\mathrm{MBC}^{\mathrm{c}}$ & - & 256 & - & - & 256 & - & - & 256 & 1 \\
\hline \multirow[t]{3}{*}{1447} & IZD & - & 10.46 & - & - & - & 7.40 & 11.81 & - & 14.19 \\
\hline & MIC & - & 1024 & - & - & - & 2048 & 512 & - & 2 \\
\hline & $\mathrm{MBC}$ & - & 2048 & - & - & - & 4096 & 1024 & - & 2 \\
\hline \multirow[t]{3}{*}{1768} & IZD & - & 15.56 & 7.24 & 8.60 & - & 7.12 & - & 7.89 & - \\
\hline & MIC & - & 256 & 1024 & 1024 & - & 1024 & - & 1024 & - \\
\hline & $\mathrm{MBC}$ & - & 512 & 2048 & 2048 & - & 2048 & - & 2048 & - \\
\hline \multirow[t]{3}{*}{1445} & IZD & - & 9.95 & - & 9.40 & 8.67 & - & - & 17.67 & 19.87 \\
\hline & MIC & - & 128 & - & 256 & 512 & - & - & 64 & 2 \\
\hline & $\mathrm{MBC}$ & - & 256 & - & 512 & 1024 & - & - & 128 & 2 \\
\hline \multirow[t]{3}{*}{1240} & IZD & - & 10.80 & - & - & - & - & - & - & 24.74 \\
\hline & MIC & - & 256 & - & - & - & - & - & - & 1 \\
\hline & $\mathrm{MBC}$ & - & 512 & - & - & - & - & - & - & 1 \\
\hline \multirow[t]{3}{*}{1297} & IZD & - & 24.12 & - & - & - & 9.67 & - & 9.90 & 18.71 \\
\hline & MIC & - & 16 & - & - & - & 1024 & - & 1024 & 2 \\
\hline & $\mathrm{MBC}$ & - & 32 & - & - & - & 2048 & - & 2048 & 2 \\
\hline \multirow[t]{3}{*}{1189} & IZD & - & 10.89 & - & - & - & - & 7.49 & - & 22.19 \\
\hline & MIC & - & 512 & - & - & - & - & 1024 & - & 1 \\
\hline & $\mathrm{MBC}$ & - & 1024 & - & - & - & - & 2048 & - & 1 \\
\hline \multirow[t]{3}{*}{1435} & IZD & 8.55 & 17.67 & 14.97 & - & 9.70 & 9.23 & - & 7.34 & 27.20 \\
\hline & MIC & 1024 & 256 & 256 & - & 512 & 512 & - & 1024 & 1 \\
\hline & $\mathrm{MBC}$ & 2048 & 512 & 512 & - & 1024 & 1024 & - & 2048 & 2 \\
\hline \multirow[t]{3}{*}{1665} & IZD & - & 17.55 & - & 8.50 & - & 8.66 & 10.56 & - & 25.51 \\
\hline & MIC & - & 128 & - & 1024 & - & 1024 & 512 & - & 0.25 \\
\hline & $\mathrm{MBC}$ & - & 256 & - & 2048 & - & 2048 & 1024 & - & 4 \\
\hline \multirow[t]{3}{*}{1023} & IZD & - & 9.11 & 7.50 & & 8.13 & 6.12 & - & 8.29 & 27.20 \\
\hline & MIC & - & 512 & 1024 & & 1024 & 1024 & - & 1024 & 1 \\
\hline & $\mathrm{MBC}$ & - & 1024 & 2048 & & 2048 & 2048 & - & 2048 & 2 \\
\hline \multirow[t]{3}{*}{1494} & IZD & - & 22.89 & - & - & - & 10.48 & - & - & 26.82 \\
\hline & MIC & - & 16 & - & - & - & 512 & - & - & 1 \\
\hline & $\mathrm{MBC}$ & - & 32 & - & - & - & 1024 & - & - & 1 \\
\hline \multirow[t]{3}{*}{1633} & IZD & - & 11.53 & - & - & - & - & - & 8.94 & 20.17 \\
\hline & MIC & - & 128 & - & - & - & - & - & 1024 & 2 \\
\hline & $\mathrm{MBC}$ & - & 256 & - & - & - & - & - & 2048 & 2 \\
\hline
\end{tabular}

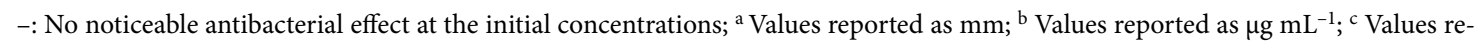
ported as $\mu \mathrm{g} \mathrm{mL} \mathrm{m}^{-1}$.

$$
\mathrm{HD}=\frac{\mathrm{LUMO}-\mathrm{HOMO}}{2} ; \mathrm{SOF}=\frac{1}{\mathrm{HD}} ; \mathrm{EN}=-\frac{\mathrm{LUMO}+\mathrm{HOMO}}{2} ; \mathrm{EPH}=-\frac{\mathrm{EN}^{2}}{2 \mathrm{HD}}
$$

Moreover, maximum and minimum local electrostatic surface potential (Max.LESP, Min.LESP) as well as average positive and average negative electrostatic potential $(\operatorname{AESP}(+), \operatorname{AESP}(-))$ were applied to characterize relatively strong dipole-dipole interactions, namely hydrogen and halogen bonds in ligand-receptor complexes. ${ }^{42}$ Furthermore, the weak intermolecular dispersion forces have been outlined by molecular volume (MV), molecular surface area (MSA), and polarizability (PL). The numerical values of the molecular descriptors were calculated and are presented in Table 4.
The information mathematical models obtained from four Gram-negative $(1855,1234,1399,1776)$ and five Gram-positive $(1447,1297,1665,1768,1435)$ bacteria are shown in Table 5. The inhibitory activity of thioethers $4 \mathbf{a}-$ $\mathbf{h}$ against Gram-negative strains were correlated to their both chemical and reversible physical descriptors, and are presented in Figures 3 and 4.

In Figure 3-A, the IZD values of compounds $\mathbf{4 b}, \mathbf{e}, \mathbf{f}, \mathbf{g}$ against bacterium 1855 were reasonably related to covalent bond descriptors including HLG (HOMO-LUMO gap), HD, SOF and EPH. This implies that covalent bonds 
Table 4. The numerical values of the calculated descriptors.

\begin{tabular}{lcccccccc}
\hline $\begin{array}{l}\text { Products } \\
\text { Descriptors }\end{array}$ & $\mathbf{4 a}$ & $\mathbf{4 b}$ & $\mathbf{4 c}$ & $\mathbf{4 d}$ & $\mathbf{4 e}$ & $\mathbf{4 f}$ & $\mathbf{4 g}$ & $\mathbf{4 h}$ \\
\hline HOMO $^{\mathrm{a}}$ & -0.2533 & -0.2561 & -0.2721 & -0.2766 & -0.2515 & -0.2570 & -0.2650 & -0.2709 \\
LUMO $^{\mathrm{a}}$ & -0.1046 & -0.1227 & -0.1281 & -0.1482 & -0.0982 & -0.1050 & -0.1123 & -0.1196 \\
$\mathrm{HLG}^{\mathrm{a}}$ & 0.1487 & 0.1334 & 0.1441 & 0.1284 & 0.1533 & 0.1520 & 0.1527 & 0.1512 \\
$\mathrm{HD}^{\mathrm{a}}$ & 0.0743 & 0.0667 & 0.0720 & 0.0642 & 0.0766 & 0.0760 & 0.0764 & 0.0756 \\
$\mathrm{SOF}^{\mathrm{b}}$ & 13.45 & 15.00 & 13.88 & 15.57 & 13.05 & 13.16 & 13.09 & 13.23 \\
$\mathrm{EN}^{\mathrm{a}}$ & -0.1790 & -0.1894 & -0.2001 & -0.2124 & -0.1748 & -0.1810 & -0.1886 & -0.1953 \\
EPH $^{\mathrm{a}}$ & -0.2155 & -0.2689 & -0.2779 & -0.3511 & -0.1994 & -0.2155 & -0.2330 & -0.2521 \\
$\mathrm{AESP}^{\mathrm{c}+)^{\mathrm{c}}}$ & 13.13 & 14.79 & 19.53 & 20.76 & 11.96 & 12.41 & 14.71 & 15.38 \\
${\mathrm{AESP}(-)^{\mathrm{c}}}^{-13.38}$ & -16.27 & -15.55 & -12.44 & -12.52 & -12.61 & -17.37 & -8.29 \\
Max.LESP $^{\mathrm{c}}$ & 32.67 & 30.56 & 42.30 & 43.18 & 31.50 & 39.62 & 33.10 & 33.77 \\
Min.LESP $^{\mathrm{c}}$ & -37.66 & -35.96 & -31.52 & -29.63 & -38.29 & -36.12 & -33.64 & -32.87 \\
MV $^{\mathrm{d}}$ & 969.0 & 1045.1 & 1078.5 & 1181.6 & 1033.0 & 1120.4 & 1021.2 & 1034.3 \\
MSA $^{\mathrm{e}}$ & 570.4 & 589.0 & 662.6 & 712.7 & 607.8 & 672.0 & 556.9 & 619.0 \\
PL $^{\mathrm{f}}$ & 37.49 & 41.17 & 41.17 & 44.60 & 41.35 & 45.20 & 42.28 & 36.58 \\
\hline
\end{tabular}

a Values reported as hartree; ${ }^{\mathrm{b}}$ Values reported as hartree ${ }^{-1} ;{ }^{\mathrm{c}}$ Values reported as kcal.mol ${ }^{-1} ;{ }^{\mathrm{d}}$ Values reported as $\AA^{3} ;{ }^{\mathrm{e}}$ Values reported as $\AA^{2}$; ${ }^{\text {f }}$ Values reported as a.u.

Table 5. The correlation coefficient $\left(r^{2}\right)$ and rate of change sign (+) or (-) of QSAR calculations.

\begin{tabular}{|c|c|c|c|c|c|c|c|c|c|}
\hline Descriptors & $\begin{array}{l}\text { Bacteria } \\
1855\end{array}$ & 1234 & 1399 & 1776 & 1447 & 1297 & 1665 & 1768 & 1435 \\
\hline HOMO & - & 0.9909: (-) & - & 0.9927: $(+)$ & - & - & - & - & - \\
\hline LUMO & - & 0.9982: (-) & - & - & - & - & 0.9540: (-) & - & - \\
\hline HLG & 0.9384: (-) & 0.9998: (-) & 0.7779: (-) & - & - & 0.9995: (-) & $0.9435:(-)$ & - & 0.8253: (-) \\
\hline $\mathrm{HD}$ & 0.9384: (-) & 0.9998: (-) & $0.7779:(-)$ & - & - & 0.9995: (-) & $0.9435:(-)$ & - & 0.8253: (-) \\
\hline SOF & $0.9362:(+)$ & 0.9999: (+) & $0.7840:(+)$ & - & - & 0.9997: (+) & $0.9456:(+)$ & - & $0.8215:(+)$ \\
\hline EN & - & 0.9963: (-) & - & - & 0.8611:(-) & - & - & - & - \\
\hline $\mathrm{EPH}$ & 0.6554: (-) & 0.9958: (-) & $0.6281:(-)$ & - & - & - & 0.9853:(-) & - & - \\
\hline $\operatorname{AESP}(+)$ & - & 0.9985: (+) & 0.6973: (+) & - & 0.8931: (+) & - & - & - & - \\
\hline $\operatorname{AESP}(-)$ & - & - & 0.8084: (-) & $0.8007:(+)$ & 0.9938: (-) & 0.6947: (-) & 0.9984: (-) & $0.7188:(-)$ & $0.7278:(-)$ \\
\hline Max.LESP & $0.7828:(+)$ & 0.9951: (+) & - & - & $0.7027:(-)$ & $0.6088:(-)$ & $0.9202:(-)$ & 0.8041: $(+)$ & - \\
\hline Min.LESP & - & 0.9990: (-) & - & 0.9854: (-) & 0.5978: (+) & - & - & - & - \\
\hline MV & $0.9647:(+)$ & - & - & - & 0.9953:(-) & - & $0.9024:(-)$ & 0.8740: (-) & - \\
\hline MSA & 0.9749: $(+)$ & - & - & - & 0.9992: (-) & 0.6159: (-) & $0.9663:(-)$ & 0.9995: (-) & - \\
\hline PL & $0.7474:(+)$ & - & - & $0.7617:(-)$ & $0.7074:(-)$ & - & 0.9311:(-) & 0.8883: (-) & - \\
\hline
\end{tabular}

are formed because of a charge transfer process from the nucleophilic centers to the electrophilic sites. Furthermore, moderate to weak correlations were observed against reversible physical descriptors (Max.LESP, MV, MSA and PL), removal of $\mathbf{4 b}$ strikingly improved them. It can be deduced that less effective ligands (molecules with lesser IZD values) preferably act via establishing dipoledipole and short distance dispersion forces. Inhibitory activity against strain 1234 was observed with all thioethers, except compound 4c. However, as indicated in Figure 3-B, their action mechanism is not the same. Different trends against irreversible covalent descriptors were found in series $\mathbf{4 b}, \mathbf{f}, \mathbf{g}, \mathbf{h}$ and $\mathbf{4 a}, \mathbf{d}, \mathbf{e}$. The former series, except ligand 4f, behaved differently from the latter one toward the dipole-dipole descriptors. No reasonable relationship was found between antibacterial activities of synthesized compounds against species 1234 and the dispersion forces de- scriptors, it reflects the dominant contribution of dipoledipole and covalent interactions. Similar weak correlations on dispersion forces descriptors were observed against bacterial species 1399 and 1776, as shown in Figure 4.

The antibacterial effects of thioethers $4 \mathbf{a}-\mathbf{h}$ on five Gram-positive strains have been correlated to their physicochemical descriptors in Figures 5 and 6. Moderate to good relationships were established between the IZD values of ligands $\mathbf{4 b}, \mathbf{f}, \mathbf{g}$ and all reversible descriptors against bacterium 1447, EN was the only irreversible parameter that can make a meaningful correlation (Figure 5-A). This probably represents a more prominent role of reversible interactions in observed effects.

The irreversible descriptors including HLG, HD and SOF show better correlations with the inhibitory properties of ligands $\mathbf{4 b}, \mathbf{f}, \mathbf{h}$ on bacterium 1297 than reversible ones including AESP(-), Max.LESP and MSA, as illustrated in 


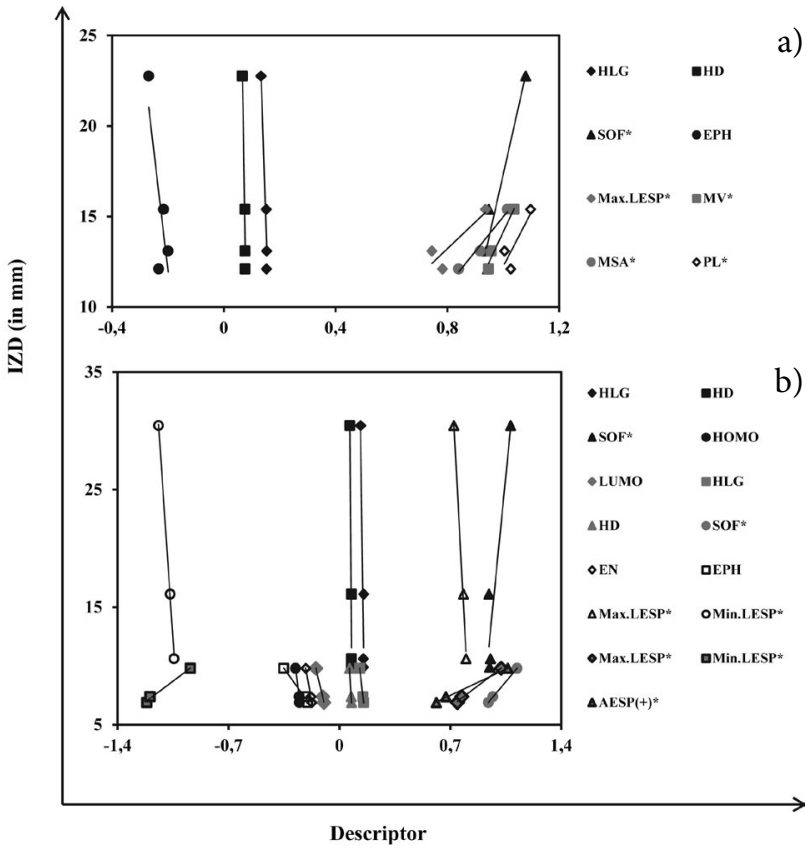

Figure 3. The correlation between descriptors and the IZD values of the ligands $\mathbf{4 b}, \mathbf{e}-\mathbf{g}$ and $\mathbf{4 a}, \mathbf{b}, \mathbf{d}-\mathbf{h}$ against Gram-negative bacteria 1855 (a) and 1234 (b), respectively. Descriptors with large outlying numerical values (superscripted by ${ }^{*}$ ) are divided by them of ligand $\mathbf{4 c}$ for the charts to be able to cover all the numbers.

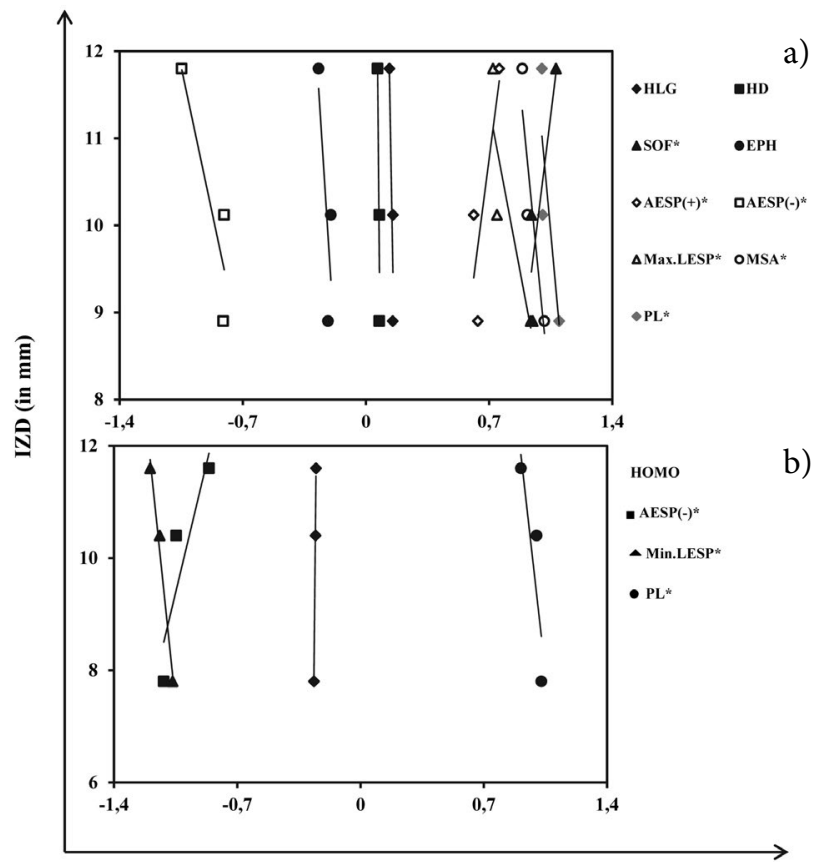

Figure 4. The correlation between descriptors and the IZD values of the ligands $\mathbf{4 b}, \mathbf{e}, \mathbf{f}$ and $\mathbf{4 a}, \mathbf{f}, \mathbf{g}$ against Gram-negative bacteria 1399 (a) and 1776 (b), respectively.

Figure 5-B. The orbital overlapping is the most probable type of interactions occurring between ligands and microorganisms. The same results can be seen in the Figure 5-C. While the antimicrobial activities of the ligands $\mathbf{4 b}, \mathbf{f}, \mathbf{g}$ on

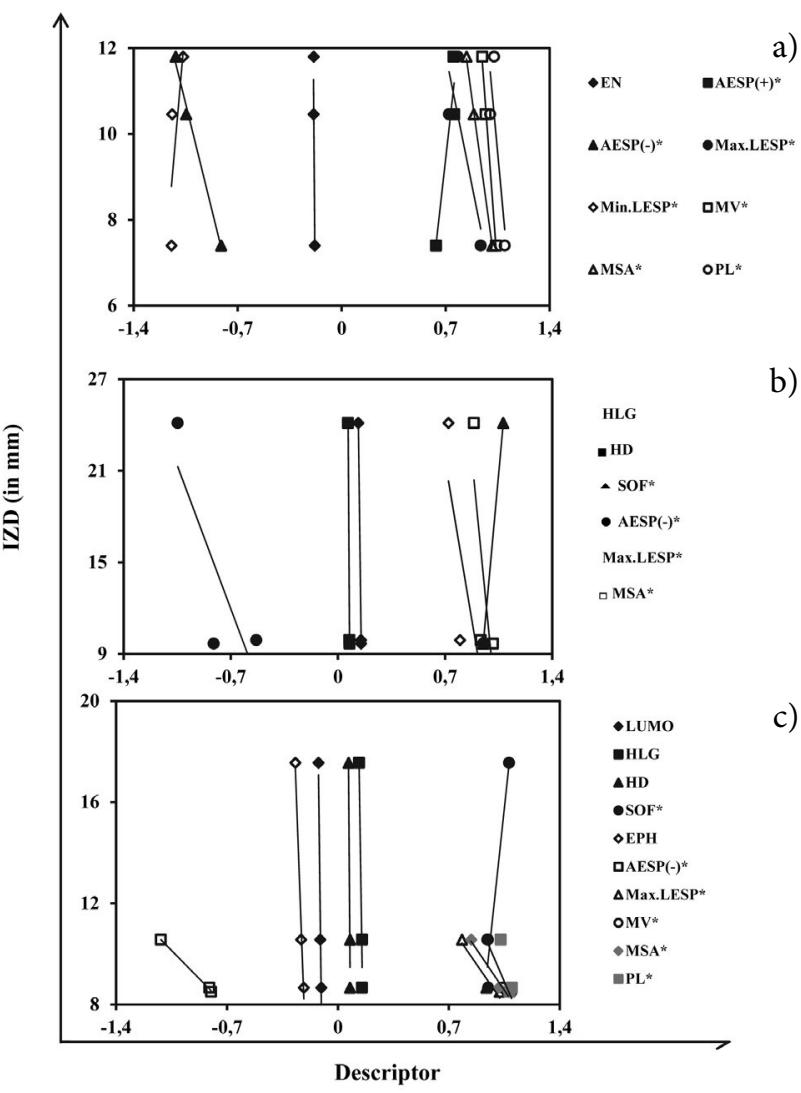

Figure 5. The correlation between descriptors and the IZD values of the ligands $\mathbf{4 b}, \mathbf{f}, \mathbf{g}$ and $\mathbf{4 b}, \mathbf{f}, \mathbf{h}$ and $\mathbf{4 b}, \mathbf{d}, \mathbf{f}, \mathbf{g}$ against Gram-positive bacteria 1447 (a), 1297 (b) and 1665 (c), respectively.

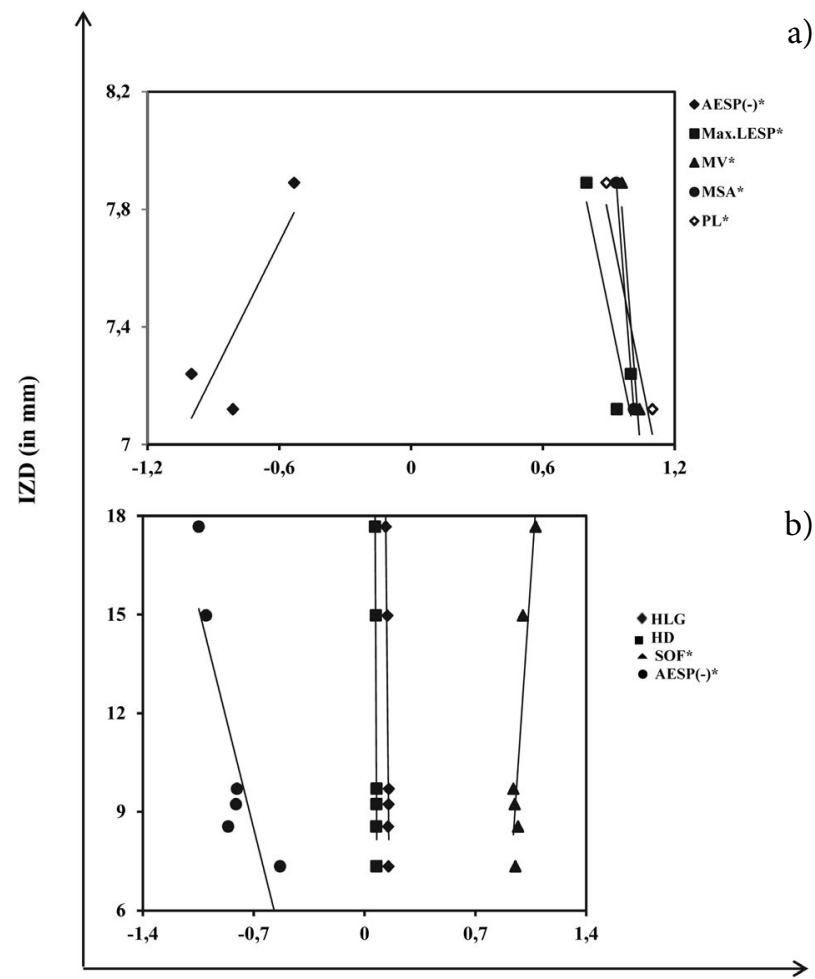

Figure 6. The correlation between descriptors and the IZD values of the ligands $\mathbf{4 c , f , h}$ and $\mathbf{4 a - c , e , f , h}$ against Gram-positive bacteria 1768 (a) and 1435 (b), respectively. 
strain 1665 have been strongly correlated to irreversible descriptors, any clear relationship was observed on the reversible dipole-dipole and London dispersion descriptors. Acceptable correlations were found with reversible interactions when compounds $\mathbf{4 d}, \mathbf{f}, \mathbf{g}$ without considering molecule $\mathbf{4 b}$ were included in calculations. This indicates that the action mechanism of the highly active ligand $\mathbf{4 b}$ against bacterium 1665 is preferably through covalent interactions. Two Gram-positive bacteria 1768 and 1435 exhibited quite different behavior. As indicated in Figure 6, while the former strain is affected by a few ligands $(\mathbf{4 c , f , h})$ with relatively low activities, all ligands, except thioether $\mathbf{4 d}$, could more efficiently inhibit the growth of the latter strain. Low activities

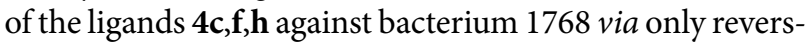
ible interactions can be seen in Figure 6-A. However, covalent descriptors such as HLG, HD and SOF were dominant interactions in thioethers $\mathbf{4 a}, \mathbf{b}, \mathbf{c}, \mathbf{e}, \mathbf{f}, \mathbf{g}, \mathbf{h}$ against bacterium 1435, as shown in Figure 6-B.

The resulting data presented in Table 5 show that the inhibitory activity of the ligands on $4 \mathrm{Gram}$-negative bacteria, was mainly related both to irreversible chemical and reversible physical (dipole-dipole and short distance dispersion forces) interactions. However, no logical correlation was observed between short distance descriptors of ligands and their antibacterial activities against microorganisms 1234 and 1399. In contrast, acceptable relationships were stablished on Gram-positive bacteria when only dipole-dipole interactions were considered. There is no such relation between the IZD values on bacteria 1768 and 1435, and covalent and London dispersion interactions, respectively. As a result, associations between Gram-positive bacterial receptors and host ligands were interpreted by dipole-dipole interactions including hydrogen and halogen bonding rather than the chemical bonding and dispersion forces. The presence or absence of interactions contributing to our QSAR calculations are summarized in Table 6.

The numerical values of some descriptors associated

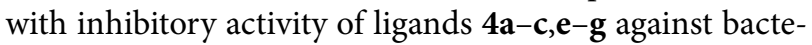
ria $1855,1234,1297,1665$ and 1435 were compared with each other to elucidate the basis for the significantly highest activity of the ligand $\mathbf{4 b}$ (Figure 7 ). Information of ligand $4 \mathbf{d}$ was not considered because of lack of its correlation with $\mathbf{4 b}$. The minimum HD, the maximum SOF and the minimum Max.LESP belonging to thioether $4 \mathrm{~b}$ is illustrated in Figure 7-A, -B and -C, respectively. As a result, it is predicted that derivative $\mathbf{4 b}$ should have the least stability and the most reactivity toward the nucleophile-electrophile chemical interactions.

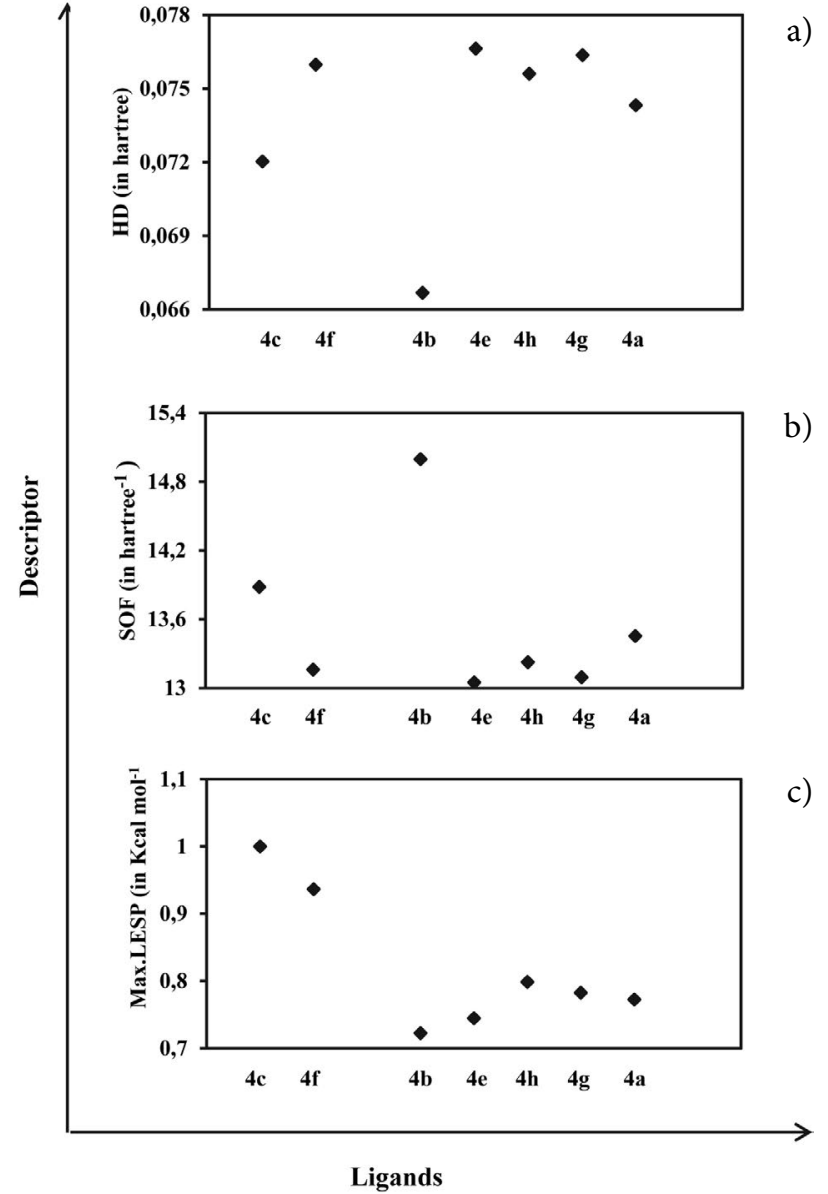

Figure 7. Comparison of HD (a), SOF (b) and Max.LESP (c) of all ligands, except $\mathbf{4 d}$, with them values of the highly active ligand $\mathbf{4 b}$.

\section{Conclusions}

Thioether derivatives $\mathbf{4 a}-\mathbf{h}$ were synthesized via two procedures. The antimicrobial activity of all synthesized ligands was evaluated against a variety of both Gram-negative and Gram-positive pathogenic bacteria. Theoretical calculations were conducted to correlate inhibitory activity of these ligands into their physico-chemical descriptors. It was found that highly active ligands could affect both Gram-positive and Gram-negative bacteria via covalent bonding interactions. Less active ligands preferably bound bacteria through both hydrogen bond and short distance London dispersion forces. Relatively strong dipole-dipole interactions of hydrogen and halogen bonding contribute in all ligand-receptor interactions. Furthermore, the same

Table 6. The presence (P) or absence (A) of interactions contributing to QSAR calculations.

\begin{tabular}{llllllllll}
\hline Interactions & \multicolumn{2}{l}{ Bacteria } & & & & & & & \\
& $\mathbf{1 8 5 5}$ & $\mathbf{1 2 3 4}$ & $\mathbf{1 3 9 9}$ & $\mathbf{1 7 7 6}$ & $\mathbf{1 4 4 7}$ & $\mathbf{1 2 9 7}$ & $\mathbf{1 6 6 5}$ & $\mathbf{1 7 6 8}$ & $\mathbf{1 4 3 5}$ \\
Covalent bonding & $\mathrm{P}$ & $\mathrm{P}$ & $\mathrm{P}$ & $\mathrm{P}$ & $\mathrm{P}$ & $\mathrm{P}$ & $\mathrm{P}$ & $\mathrm{A}$ & $\mathrm{P}$ \\
Dipole-dipole & $\mathrm{P}$ & $\mathrm{P}$ & $\mathrm{P}$ & $\mathrm{P}$ & $\mathrm{P}$ & $\mathrm{P}$ & $\mathrm{P}$ & $\mathrm{P}$ & $\mathrm{P}$ \\
Dispersion forces & $\mathrm{P}$ & $\mathrm{A}$ & $\mathrm{A}$ & $\mathrm{P}$ & $\mathrm{P}$ & $\mathrm{P}$ & $\mathrm{P}$ & $\mathrm{P}$ & $\mathrm{A}$ \\
\hline
\end{tabular}


rate of change of sign was observed at both classes of bacteria for irreversible covalent descriptors (Table 5). However, signs in descriptors including Max.LESP, Min.LESP, MV, MSA and PL were opposite to each other. These models convey that calculations must be focused on short distance dispersion forces and dipole-dipole interactions rather than covalent bonding to find differences in the mechanisms of action of ligands against Gram-negative and Gram-positive bacteria. The models propose that high softness of ligand $\mathbf{4 b}$ is probably the main cause of its widespread and strong antibacterial activities. Indeed, it efficiently links to targets via chemical covalent bonding.

\section{Acknowledgements}

This work was supported by the University of Zabol under Grant number UOZ-GR-9517-15.

\section{References}

1. Y. Sanada, H. Senba, R. Mochizuki, H. Arakaki, T. Gotoh, S. Fukumoto, H. Nagahata, J. Vet. Med. Sci. 2009, 71, 617-620. DOI:10.1292/jvms.71.617

2. W. Leonardi, L. Zilbermintz, L. W. Cheng, J. Zozaya, S. H. Tran, J. H. Elliott, K. Polukhina, R. Manasherob, A. Li, X. Chi, D. Gharaibeh, T. Kenny, R. Zamani, V. Soloveva, A. D. Haddow, F. Nasar, S. Bavari, M. C. Bassik, S. N. Cohen, A. Levitin, M. Martchenko, Sci. Rep. 2016, 6, 34475. DOI:10.1038/srep34475

3. R. Muller, C. Bottger, G. Wichmann, Arzneimittelforschung 2003, 53, 126-132. DOI:10.1055/s-0031-1297083

4. L. S. Seidman, C. K. Skokos, Infect. Dis. Obstet. Gynecol. 2005, 13, 197-206. DOI:10.1155/2005/453239

5. H. Wu, Y. Zhang, J. Huang, Y. Zhang, G. Liu, N. Sun, Z. Yu, Y. Zhou, Hypertens. Res. 2001, 24, 605-610. DOI:10.1291/hypres.24.605

6. K. -S. Lee, J. -S. Kim, J. -W. Kim, W. -Y. Lee, B. -S. Jeon, D. Kim, Parkinsonism Relat. Disord. 2003, 9, 341-347. DOI:10.1016/S1353-8020(03)00029-4

7. S. Yamashita, Y. Matsuzawa, atherosclerosis 2009, 207, 16-23. DOI:10.1016/j.atherosclerosis.2009.04.002

8. A. Esposito, M. R. Valentino, D. Bruzzese, M. Bocchino, A. Ponticiello, A. Stanziola, A. Sanduzzi, Pulm. Pharmacol. Ther. 2016, 37, 85-88. DOI:10.1016/j.pupt.2016.03.003

9. M. S. Oderinde, M. Frenette, D. W. Robbins, B. Aquila, J. W. Johannes, J. Am. Chem. Soc. 2016, 138, 1760-1763. DOI:10.1021/jacs.5b11244

10. M. Jouffroy, C. B. Kelly, G. A. Molander, Org. Lett. 2016, 18, 876-879. DOI:10.1021/acs.orglett.6b00208

11. F. Santoro, M. Mariani, F. Zaccheria, R. Psaro, N. Ravasio, Beilstein J. Org. Chem. 2016, 12, 2627-2635. DOI:10.3762/bjoc.12.259

12. Y. Li, J. Pu, X. Jiang, Org. Lett. 2014, 16, 2692-2695. DOI:10.1021/ol5009747
13. S. M. M. Elshafie, Org. Prep. Proc. Int. 1983, 15, 225-231. DOI:10.1080/00304948309356646

14. J.-H. Cheng, C. Ramesh, H.-L. Kao, Y.-J. Wang, C.-C. Chan, C.-F. Lee, J. Org. Chem. 2012, 77, 10369-10374. DOI:10.1021/jo302088t

15. O. O. Fadeyi, J. J. Mousseau, Y. Feng, C. Allais, P. Nuhant, M. Z. Chen, B. Pierce, R. Robinson, Org. Lett. 2015, 17, 57565759. DOI:10.1021/acs.orglett.5b03184

16. V. J. Gray, J. Cuthbertson, J. D. Wilden, J. Org. Chem. 2014, 79, 5869-5874. DOI:10.1021/jo500814y

17. S. Shaw, J. D. White, Org. Lett. 2015, 17, 4564-4567. DOI:10.1021/acs.orglett.5b02280

18. G. A. Molander, J. Ham, Org. Lett. 2006, 8, 2031-2034. DOI:10.1021/ol060375a

19. G. Turkoglua, M. E. Cinar, T. Ozturk, Synthesis 2016, 48, 3618-3624. DOI:10.1055/s-0035-1561673

20. X.-B. Xu, Z.-H. Lin, Y. Liu, J. Guo, Y. He, Org. Biomol. Chem. 2017, 15, 2716-2720. DOI:10.1039/C7OB00277G

21. N. Azizi, Z. Yadollahy, A. Rahimzadeh-Oskooee, Synlett 2014, 25, 10851088. DOI:10.1055/s-0033-1341050

22. Y. Nishimoto, A. Okita, M. Yasuda, A. Baba, Org. Lett. 2012, 14, 1846-1849. DOI:10.1021/ol300450j

23. N. Sakai, T. Miyazaki, T. Sakamoto, T. Yatsuda, T. Moriya, R. Ikeda, T. Konakahara, Org. Lett. 2012, 14, 4366-4369. DOI:10.1021/ol302109v

24. V. Padmavathi, G. Dinneswara Reddy, S. Nagi Reddy, K. Mahesh, Eur. J. Med. Chem. 2011, 46, 1367-1373.

DOI:10.1016/j.ejmech.2011.01.063

25. Q. Huang, P. F. Richardson, N. W. Sach, J. Zhu, K. K. -C. Liu, G. L. Smith, D. M. Bowles, Org. Process Res. Dev. 2011, 15, 556-564. DOI:10.1021/op100286g

26. I. Yavari, E. Sodagar, M. Nematpour, Helv. Chim. Acta 2014, 97, 420-425. DOI:10.1002/hlca.201300345

27. G. C. Patra, S. Pal, S. C. Bhunia, N. K. Hazra, S. C. Pal, Indian J. Chem. 2016, 55B, 471-477.

28. V. D. Dyachenko, V. N. Nesterov, I. V. Dyachenko, Russ. J. Gen. Chem. 2011, 81, 751-755.

DOI:10.1134/S1070363211040232

29. A. Sadeghian, S. M. Seyedi, H. Sadeghian, A. Hazrathoseyni, M. Sadeghian, J. Sulfur Chem. 2007, 28, 597-605. DOI:10.1080/17415990701670718

30. Z. Eskandariyan, M. Esfahanizadeh, K. Haj Mohammad Ebrahim Tehrani, V. Mashayekhi, F. Kobarfard, Arch. Pharmacal Res. 2014, 37, 332-339.

DOI:10.1007/s12272-013-0192-5

31. Z. Wang, Z. Wang, L. Ping Cheng, Lett. Drug Des. Discovery $2017,14,869-879$.

DOI:10.2174/1570180814666170111154331

32. J. J. D’amico, P. G. Ruminski, L. A. Suba, J. J. Freeman, W. E. Dahl, Phosphorus Sulfur Relat. Elem. 1985, 21, 307-314. DOI:10.1080/03086648508077673

33. H. Beyzaei, Z. Motraghi, R. Aryan, B. Ghasemi, M. M. Zahedi, A. Samzadeh-Kermani, Acta Chim. Slov. 2017, 64, 911918. DOI:10.17344/acsi.2017.3609

34. M. J. Frisch, G. W. Trucks, H. B. Schlegel, G. E. Scuseria, M. A. Robb, J. R. Cheeseman, G. Scalmani, V. Barone, B. Men- 
nucci, G. A. Petersson, H. Nakatsuji, M. Caricato, X. Li, H. P. Hratchian, A. F. Izmaylov, J. Bloino, G. Zheng, J. L. Sonnenberg, M. Hada, M. Ehara, K. Toyota, R. Fukuda, J. Hasegawa, M. Ishida, T. Nakajima, Y. Honda, O. Kitao, H. Nakai, T. Vreven, J. A. Montgomery, J. E. Peralta, F. Ogliaro, M. Bearpark, J. J. Heyd, E. Brothers, K. N. Kudin, V. N. Staroverov, R. Kobayashi, J. Normand, K. Raghavachari, A. Rendell, J. C. Burant, S. S. Iyengar, J. Tomasi, M. Cossi, N. Rega, J. M. Millam, M. Klene, J. E. Knox, J. B. Cross, V. Bakken, C. Adamo, J. Jaramillo, R. Gomperts, R. E. Stratmann, O. Yazyev, A. J. Austin, R. Cammi, C. Pomelli, J. W. Ochterski, R. L. Martin, K. Morokuma, V. G. Zakrzewski, G. A. Voth, P. Salvador, J. J. Dannenberg, S. Dapprich, A. D. Daniels, O. Farkas, J. B. Foresman, J. V. Ortiz, J. Cioslowski, D. J. Fox, Gaussian 09, Revision A 02, Gaussian, Inc., Wallingford CT, USA, 2009.

35. R. F. W. Bader, Atoms in Molecules - A Quantum Theory, 1nd ed., Oxford University Press, Oxford, England, 1990.
36. E. D. Glendening, A. E. Reed, J. E. Carpenter, F. Weinhold, NBO 3.1, Gaussian, Inc., Pittsburgh PA, USA, 2001.

37. R. M. LoPachin, D. S. Barber, T. Gavin, Toxicol. Sci. 2008, 104, 235-249. DOI:10.1093/toxsci/kfm301

38. R. M. LoPachin, T. Gavin, D. R. Petersen, D. S. Barber, Chem. Res. Toxicol. 2009, 22, 1499-1508. DOI:10.1021/tx900147g

39. R. G. Pearson, Coord. Chem. Rev. 1990, 100, 403-425. DOI:10.1016/0010-8545(90)85016-L

40. R. G. Parr, P. K. Chattaraj, J. Am. Chem. Soc. 1991, 113, 18541855. DOI:10.1021/ja00005a072

41. P. Thanikaivelan, V. Subramanian, J. R. Rao, B. U. Nair, Chem. Phys. Lett. 2000, 323, 59-70.

DOI:10.1016/S0009-2614(00)00488-7

42. F. A. Bulat, A. Toro-Labbé, T. Brinck, J. S. Murray, P. Politzer, J. Mol. Model. 2010, 16, 1679-1691. DOI:10.1007/s00894-010-0692-X

\section{Povzetek}

Razvili smo večkomponentno reakcijo malononitrila, ogljikovega disulfida in različnih benzil halidov ter jo uporabili kot učinkovito strategijo za sintezo serije 2-(bis(benziltio)metilen)malononitrilnih derivatov. Sinteze smo izvedli na dva načina: (a) v prisotnosti $\mathrm{K}_{2} \mathrm{CO}_{3}$ kot baze $\mathrm{v}$ acetonitrilu in (b) pod pogoji brez topil v prisotnosti trietilamina. Višji izkoristki in krajši reakcijski časi so bili odlika pristopa (b). Inhibitorne aktivnosti vseh spojin smo ugotovili za 22 različnih patogenih bakterij, ki so vključevale tako Gram-negativne kot tudi Gram-pozitivne seve. Tioeter $\mathbf{4 b}$ je v antibiogramskih testih izkazal široko antibakterijsko aktivnost. Da bi ugotovili vrsto interakcij med učinkovinami in receptorji, smo izvedli tudi DFT izračune (B3LYP/6-311++ $\mathrm{G}^{* *}$ ); izkazalo se je, da reverzibilne dipol-dipol interakcije v večini primerov igrajo ključno vlogo. 
